\title{
Dietary learning: both consistency and congruency matter
}

Paul A.M. Smeets

Recently, a Perspectives article by De Graaf \& Kok (Slow food, fast food and the control of food intake. Nat. Rev. Endocrinol. 6, 290-293; 2010) $)^{1}$ hypothesized that a high eating rate and a lack of attention for the act of eating disrupt the regulation of energy intake "because the congruent relationship between sensory input and metabolic consequences is impaired". Later on they conclude that these two factors "do this by lowering the consistency of the relationship" between sensory signals and ensuing metabolic consequences. However, congruency and consistency are different things. For example, an incongruent combination of sweet taste without caloric content, as found in 'light' drinks, could still be consumed very consistently. Consistency will facilitate flavor-nutrient learning, also in the case of incongruent food stimuli. Important issues that have not yet been elucidated satisfactorily are to what extent incongruent combinations of sensory characteristics and caloric content, which are very common, disrupt physiological processes $^{2}$ and to what extent this disruption is aggravated by inconsistency, as in the common case of consumption of both 'light' and 'regular' foods. In short, it is crucial to assess the respective effects of congruency and consistency as well as their interaction.
Further, De Graaf \& Kok point out, in line with the 'thrifty gene' hypothesis, ${ }^{3}$ that the obesity epidemic is a normal behavioral response to an abnormal obesogenic environment. From the perspective of a 'thrifty genotype', fast foods, which are readily available, cheap and energy-dense, are 'smart foods'. Such foods should become preferred, and they do so by way of flavor-nutrient learning, which enables animals to estimate the caloric content of a food based on previous experience with that food. ${ }^{4,5}$ Thus, the primary function of food-related sensory signals (e.g., visual, gustatory, olfactory) is identification, whereas quantification of ingested calories occurs on the basis of metabolic signals, which are far more reliable indicators of caloric content than sensory signals. ${ }^{6}$ The latter is not in accordance with De Graaf \& Kok's hypothesis that a 'mismatch' between the amount of oro-sensory stimulation and subsequent metabolic consequences may fool the body. This suggests that not just the type, but also the amount of sensory stimulation is used to predict upcoming metabolic consequences. In addition, the mere fact that ingested calories are not compensated for later on by decreased energy intake, even after repeated consumption, does not prove unambiguously that these calories go 'unnoticed'. The data cited by De Graaf \& Kok can also be interpreted as a tendency to maximize (the rate of) energy intake within the constraints posed by a food's physical properties. Such a trait could very well have been favored by natural selection, as part of a 'thrifty genotype', and is indeed detrimental for long-term health in our current food environment. ${ }^{3}$

Finally, the protein-leverage hypothesis, ${ }^{7}$ which postulates that natural selection did not favor maximization of energy intake/ fat stores but rather an optimization of protein intake, is a noteworthy complementary hypothesis because it suggests that overeating could be suppressed by altering the macronutrient composition of our diet.

University Medical Center Utrecht, Heidelberglaan 100, Room Q0S.459, 3584 CX Utrecht, The Netherlands paul@isi.uu.nl

doi:10.1038/nrendo.2010.41-c1

Competing interests

The author declares no competing interests.

1. De Graaf, C. and Kok, F. J. Slow food, fast food and the control of food intake. Nat. Rev. Endocrinol. 6, 290-293 (2010).

2. Swithers, S. E., Martin, A. A. \& Davidson, T. L. High-intensity sweeteners and energy balance. Physiol. Behav. 100, 55-64 (2010).

3. Neel, J. V. Diabetes mellitus: "thrifty" genotype rendered detrimental by "progress"? Am. J. Hum. Genetics 14, 353-362 (1962).

4. Sclafani, A. Learned controls of ingestive behaviour. Appetite 29, 153-158 (1997).

5. Brunstrom, J. M. Does dietary learning occur outside awareness? Conscious. Cogn. 13, 453-470 (2004).

6. Woods, S. C. The control of food intake: behavioral versus molecular perspectives. Cell Metab. 9, 489-498 (2009).

7. Simpson, S. J. \& Raubenheimer, D. Obesity: the protein leverage hypothesis. Obes. Rev. 6 , 133-142 (2005). 